\title{
Ultracold atomic collisions in tight harmonic traps: Perturbation theory, ionization losses and application to metastable helium atoms
}

\author{
T J Beams†, G Peach $\ddagger$ and I B Whittingham $\dagger$ \\ † School of Mathematical and Physical Sciences, James Cook University, Townsville, \\ Australia, 4811 \\ $\ddagger$ Department of Physics and Astronomy, University College London, Gower Street, \\ London, WC1E 6BT, UK
}

\begin{abstract}
Collisions between tightly confined atoms can lead to ionization and hence to loss of atoms from the trap. We develop second-order perturbation theory for a tensorial perturbation of a spherically symmetric system and the theory is then applied to processes mediated by the spin-dipole interaction. Redistribution and loss mechanisms are studied for the case of spin-polarized metastable helium atoms and results obtained for the five lowest $s$ states in the trap and trapping frequencies ranging from $1 \mathrm{kHz}$ to $10 \mathrm{MHz}$.
\end{abstract}

PACS numbers: 32.80.Pj, 32.80.Dz, 34.20.Cf 


\section{Introduction}

There is significant interest in the study and control of quantum processes involving trapped ultracold neutral atoms where the trapping environments are so tight that the effect of the trapping fields upon the colliding atoms cannot be ignored or approximated as constant background fields. Trapping in three-dimensional optical lattices, with typical trapping frequencies of $10^{4}$ to $10^{6} \mathrm{~Hz}$, forms the basis of such studies as quantum phase transitions of ${ }^{87} \mathrm{Rb}$ atoms [1], storage of metastable argon atoms [2], implementation of quantum logic gates and formation of highly entangled quantum states 3, 4]. Theoretical investigations have focussed mainly on tightly confined alkali systems and have been based either upon direct numerical integration of the radial Schrödinger equation for the relative motion of the colliding atoms using the best available full interatomic potentials [5] or upon a regularized delta-function pseudopotential and an energy-dependent effective scattering length [6, 7].

An understanding of collision processes in trapped ultracold metastable $2^{3} \mathrm{~S}$ helium (denoted by $\mathrm{He}^{*}$ ) is necessary to obtain Bose-Einstein condensation of this species [8, 9] and to investigate these novel excited-state condensates [10, 11. Although current experiments on $\mathrm{He}^{*}$ only use trapping frequencies of the order of $10^{2}$ to $10^{3} \mathrm{~Hz}$, it is of interest to investigate the effects of much tighter trapping on the allowed quantized trap states, as a possible tool to manipulate the confined atoms, and to enhance trap loss through ionization processes at small interatomic separations as a means of studying these processes.

We have recently analyzed a system of two colliding ultracold atoms under strong harmonic confinement in a spherically symmetric trap from the viewpoints of quantum defect theory and of elastic scattering in the interatomic potential. We have developed methods for determining the energies of the quantized states produced by the presence of the trap and the theory was applied to collisions between spin-polarized He* atoms, see Peach et al [12, 13. The energies were determined for a wide range of trapping frequencies for $s$ - and $d$ - wave collisions using two totally independent methods to integrate the radial Schödinger equation. Excellent agreement was obtained between the two methods, one based on the use of quantum defect theory and the second on the use of a discrete variable representation.

These calculations ignored loss processes, but inelastic collisions may cause transitions to states from which there is a high probability of Penning and associative ionization. A study of such loss processes is the subject of this paper which is organized as follows. In section 2, the theory of collisions in an isotropic trap is briefly reviewed, and in section 3, second-order perturbation theory is introduced for a general form of the perturbation and for trap states of any angular momentum. In section 4 , the theory is applied to perturbation by the spin-dipole interaction and in section 5 the numerical methods are described. Finally in section 6 the theory is applied to the case of spinpolarized $\mathrm{He}^{*}$ atoms. It is found that only the $s$ states are significantly perturbed and shifts and lifetimes are presented for the five lowest $s$ states in the trap for trapping 
frequencies ranging from $1 \mathrm{kHz}$ to $10 \mathrm{MHz}$.

\section{Collisions between two atoms in an isotropic harmonic trap}

Consider two atoms with masses $M_{1}$ and $M_{2}$, spin quantum numbers $S_{1}$ and $S_{2}$ and position vectors $\boldsymbol{r}_{1}$ and $\boldsymbol{r}_{2}$ relative to the centre of the trap. The interatomic separation is given by $r=|\boldsymbol{r}|=\left|\boldsymbol{r}_{1}-\boldsymbol{r}_{2}\right|$ and, for the case of an atom-atom potential that is only a function of $r$ combined with a potential for an isotropic harmonic trap of angular frequency $\omega$, the Hamiltonian is separable into two parts $H_{\mathrm{cm}}$ and $H_{0}$ describing the centre-of-mass and relative motions of the two atoms. Here we will use and extend the notation developed in [13. If the total spin quantum number is $S$ and the adiabatic potential for the molecular state ${ }^{2 S+1} \Lambda$ is denoted by $V_{\Lambda S}(r)$, the equation for the relative motion is

$$
H_{0}|\psi(\boldsymbol{r})\rangle=E|\psi(\boldsymbol{r})\rangle,
$$

where $H_{0}$ is defined by

$$
H_{0} \equiv\left[-\frac{\hbar^{2}}{2 M} \nabla_{r}^{2}+\frac{1}{2} M \omega^{2} r^{2} \Delta_{\text {trap }}+V_{\Lambda S}(r)\right] .
$$

In (11) and (2) $E$ is the energy eigenvalue, the reduced mass $M=M_{1} M_{2} /\left(M_{1}+M_{2}\right)$ and $\Delta_{\text {trap }}=1$ or 0 according to whether the harmonic potential is turned on or off. If the angular momentum quantum number for the relative motion is $l$, the eigenvector $|\psi(\boldsymbol{r})\rangle$ is given by

$$
|\psi(\boldsymbol{r})\rangle=\frac{1}{r} F_{k l \Lambda S}(r)|\operatorname{lm}\rangle\left|S_{1} S_{2} S M_{S}\right\rangle,
$$

where the magnetic quantum numbers $m$ and $M_{S}$ refer to projections of the angular and spin momenta onto the molecular axis. The radial function $F_{k l \Lambda S}(r)$ satisfies the equation

$$
\left[\frac{\mathrm{d}^{2}}{\mathrm{~d} r^{2}}-\frac{l(l+1)}{r^{2}}-\frac{r^{2}}{\xi^{4}} \Delta_{\text {trap }}-\frac{2 M}{\hbar^{2}} V_{\Lambda S}(r)+k^{2}\right] F_{k l \Lambda S}(r)=0,
$$

where we have introduced the quantities

$$
k^{2}=\frac{2 M E}{\hbar^{2}} ; \quad \xi^{2}=\frac{\hbar}{M \omega} .
$$

It has also been shown in [13] that for the discrete states with $E>0$ produced by the presence of the trap, hereafter referred to as trap states, it is natural to introduce an effective quantum number (or scaled energy) $n^{*}$, where

$$
n^{*}=\frac{E}{2 \hbar \omega}=n_{r}^{\prime}+\frac{l}{2}+\frac{3}{4}-\mu^{\prime} ; \quad n_{r}^{\prime}=0,1,2, \ldots,
$$

and $\mu^{\prime}$ is a quantum defect that varies slowly as $n_{r}^{\prime}$ increases. By introducing the dimensionless variable $\rho=r / \xi$, equation (4) can also be written in the form

$$
\left[\frac{\mathrm{d}^{2}}{\mathrm{~d} \rho^{2}}-\frac{l(l+1)}{\rho^{2}}-\rho^{2} \Delta_{\text {trap }}-\frac{2 V_{\Lambda S}(\rho)}{\hbar \omega}+4 n^{*}\right] F_{k l \Lambda S}(\rho)=0 .
$$




\section{Perturbation theory}

The Hamiltonian for the perturbed system is given by

$$
H=H_{0}+H_{\mathrm{p}}
$$

where $H_{\mathrm{p}}$ is the perturbing potential and it is assumed that the eigenstates of $H_{0}$ are known, i.e.

$$
H_{0}|j\rangle=E_{j}|j\rangle ; \quad j=0,1,2, \ldots
$$

Then the change in energy of the initial state $i$, correct to second order, is given by

$$
\Delta E=\Delta E_{1}+\Delta E_{2}
$$

where

$$
\Delta E_{1}=\left\langle i\left|H_{\mathrm{p}}\right| i\right\rangle
$$

and

$$
\Delta E_{2}=-\sum_{j \neq i} \frac{\left\langle i\left|H_{\mathrm{p}}^{\dagger}\right| j\right\rangle\left\langle j\left|H_{\mathrm{p}}\right| i\right\rangle}{\left(E_{j}-E_{i}\right)} .
$$

The calculation of $\Delta E_{1}$ is straightforward and $\Delta E_{2}$ can be evaluated as follows. Using the method of Dalgarno and Lewis [14], we introduce the operator $\hat{F}$ which satisfies the inhomogeneous equation

$$
\left[\hat{F} H_{0}-H_{0} \hat{F}\right]|i\rangle=H_{\mathrm{p}}|i\rangle
$$

so that (12) becomes

$$
\Delta E_{2}=\left\langle i\left|H_{\mathrm{p}}^{\dagger} \hat{F}\right| i\right\rangle-\left\langle i\left|H_{\mathrm{p}}^{\dagger}\right| i\right\rangle\langle i|\hat{F}| i\rangle .
$$

This result is only useful if we can determine the operator $\hat{F}$. We consider the perturbation $H_{\mathrm{p}}$ given by

$$
H_{\mathrm{p}}=\mathbf{T}(\lambda) \cdot \mathbf{U}(\lambda) V_{\mathrm{p}}(r),
$$

where $\mathbf{T}(\lambda)$ and $\mathbf{U}(\lambda)$ are tensor operators of order $\lambda$ and $V_{\mathrm{p}}(r)$ contains the radial dependence of $H_{\mathrm{p}}$. We set $|i\rangle \equiv|\psi(\boldsymbol{r})\rangle$ and make the following expansion

$\hat{F}|i\rangle \equiv \hat{F} \frac{1}{r} F_{k l \Lambda S}(r)|l m\rangle\left|S M_{S}\right\rangle=\sum_{l^{\prime} m^{\prime} S^{\prime} M_{S}^{\prime}} \frac{1}{r} f_{l^{\prime} m^{\prime} S^{\prime} M_{S}^{\prime}}(r)\left|l^{\prime} m^{\prime}\right\rangle\left|S^{\prime} M_{S}^{\prime}\right\rangle$,

where we have suppressed the spin quantum numbers $S_{1}$ and $S_{2}$ in (3). Then, on using (11) - (41), (13), (15) and (16), we obtain

$$
\begin{aligned}
& \left(\hat{F} H_{0}-H_{0} \hat{F}\right)|i\rangle=\frac{\hbar^{2}}{2 M} \sum_{l^{\prime} m^{\prime} S^{\prime} M_{S}^{\prime}} \\
& \times\left[\frac{\mathrm{d}^{2}}{\mathrm{~d} r^{2}}-\frac{l^{\prime}\left(l^{\prime}+1\right)}{r^{2}}-\frac{r^{2}}{\xi^{4}} \Delta_{\text {trap }}-\frac{2 M}{\hbar^{2}} V_{\Lambda^{\prime} S^{\prime}}+k^{2}\right] f_{l^{\prime} m^{\prime} S^{\prime} M_{S}^{\prime}}\left|l^{\prime} m^{\prime}\right\rangle\left|S^{\prime} M_{S}^{\prime}\right\rangle \\
& =\mathbf{T}(\lambda) \cdot \mathbf{U}(\lambda) V_{\mathrm{p}} F_{k l \Lambda S}|l m\rangle\left|S M_{S}\right\rangle .
\end{aligned}
$$


Now if we define $G_{k l^{\prime} \Lambda^{\prime} S^{\prime}}(r)$ by the relation

$$
f_{l^{\prime} m^{\prime} S^{\prime} M_{S}^{\prime}}(r)=\left\langle S^{\prime} M_{S}^{\prime}|\mathbf{T}(\lambda)| S M_{S}\right\rangle \cdot\left\langle l^{\prime} m^{\prime}|\mathbf{U}(\lambda)| l m\right\rangle G_{k l^{\prime} \Lambda^{\prime} S^{\prime}}(r),
$$

then $G_{k l^{\prime} \Lambda^{\prime} S^{\prime}}(r)$ satisfies the inhomogeneous radial equation

$$
\left[\frac{\mathrm{d}^{2}}{\mathrm{~d} r^{2}}-\frac{l^{\prime}\left(l^{\prime}+1\right)}{r^{2}}-\frac{r^{2}}{\xi^{4}} \Delta_{\text {trap }}-\frac{2 M}{\hbar^{2}} V_{\Lambda^{\prime} S^{\prime}}(r)+k^{2}\right] G_{k l^{\prime} \Lambda^{\prime} S^{\prime}}(r)=\frac{2 M}{\hbar^{2}} V_{\mathrm{p}}(r) F_{k l \Lambda S}(r),
$$

c.f. (44). On introducing the scaled variable $\rho$, (19) becomes

$$
\begin{aligned}
& {\left[\frac{\mathrm{d}^{2}}{\mathrm{~d} \rho^{2}}-\frac{l^{\prime}\left(l^{\prime}+1\right)}{\rho^{2}}-\rho^{2} \Delta_{\text {trap }}-\frac{2 V_{\Lambda^{\prime} S^{\prime}}(\rho)}{\hbar \omega}+4 n^{*}\right] G_{k l^{\prime} \Lambda^{\prime} S^{\prime}}(\rho)=\frac{2 V_{\mathrm{p}}(\rho)}{\hbar \omega} F_{k l \Lambda S}(\rho)} \\
& \equiv W(\rho) F_{k l \Lambda S}(\rho),
\end{aligned}
$$

c.f. (17). If $\Delta_{\text {trap }}=0$ in (44), then in the outer region where $V_{\Lambda S}(r)$ is very small, the regular and irregular solutions for $F_{k l \Lambda S}(r)$ are given by

$$
\begin{aligned}
& F_{l}^{\mathrm{R}}(r) \simeq N(k r)\left[\cos \delta_{l} j_{l}(k r)-\sin \delta_{l} n_{l}(k r)\right] ; \\
& F_{l}^{\mathrm{I}}(r) \simeq-N(k r)\left[\sin \delta_{l} j_{l}(k r)+\cos \delta_{l} n_{l}(k r)\right],
\end{aligned}
$$

where $j_{l}(k r)$ and $n_{l}(k r)$ are spherical Bessel functions, see [15], $N$ is a normalization constant and $\delta_{l} \equiv \delta_{l}(k)$ is the $l$-wave phase shift for elastic scattering. The wave function $F_{k l \Lambda S}(r)$ is matched to a normalized bound-state wave function of the same energy by choosing

$$
N=2\left[\pi \xi^{2} k\left(1+\frac{\mathrm{d} \mu^{\prime}}{\mathrm{d} n^{*}}\right)\right]^{-\frac{1}{2}},
$$

c.f. (6), where $\mu^{\prime} \equiv \mu^{\prime}\left(n^{*}\right)$ is treated as a continuous function. Also, as $r \rightarrow \infty$, it can be shown that the contribution to the solution of (19) from the particular integral is given by

$$
G_{k l^{\prime} \Lambda^{\prime} S^{\prime}}=\Re\left\{\left[F_{l}^{\mathrm{R}}(r)+\mathrm{i} F_{l}^{\mathrm{I}}(r)\right] \frac{1}{r^{2}} R(x)\right\} ; \quad x \equiv \frac{1}{r},
$$

where $R(x)$ is a slowly varying complex function of $x$ satisfying the conditions

$$
R(0)=\frac{M}{2 k} \mathrm{i} ;\left.\quad \frac{\mathrm{d} R}{\mathrm{~d} x}\right|_{x=0}=0 .
$$

In what follows we use the results in (14) - (20), average over initial degenerate states $|l m\rangle$ and carry out some angular algebra, more details of which are given in the Appendix. We introduce reduced matrix elements $\left\langle j^{\prime}\|\mathbf{X}(\lambda)\| j\right\rangle$, see (A.1) and then $\Delta E_{1}$ in (11) is given by

$\Delta E_{1}=[(2 l+1)(2 S+1)]^{-\frac{1}{2}}\langle S|| \mathbf{T}(\lambda) \| S\rangle\langle l|| \mathbf{U}(\lambda) \| l\rangle \int_{0}^{\infty} F_{k l \Lambda S}(r) V_{\mathrm{p}}(r) F_{k l \Lambda S}(r) \mathrm{d} r$

for $\lambda=0$ and zero otherwise. On using (17), (A.1) and (A.2), $\Delta E_{2}$ in (14) becomes $\Delta E_{2}=[(2 l+1)(2 \lambda+1)(2 S+1)]^{-1} \sum_{l^{\prime} S^{\prime}}\left|\left\langle S^{\prime}|| \mathbf{T}(\lambda)|| S\right\rangle\right|^{2}\left|\left\langle l^{\prime}|| \mathbf{U}(\lambda) \| l\right\rangle\right|^{2}$ 


$$
\begin{aligned}
& \times\left[\int_{0}^{\infty} G_{k l^{\prime} \Lambda^{\prime} S^{\prime}}(r) V_{\mathrm{p}}(r) F_{k l \Lambda S}(r) \mathrm{d} r\right. \\
& \left.-\delta_{l^{\prime} l} \delta_{S^{\prime} S} \int_{0}^{\infty} F_{k l \Lambda S}(r) V_{\mathrm{p}}(r) F_{k l \Lambda S}(r) \mathrm{d} r \int_{0}^{\infty} G_{k l \Lambda S}(r) F_{k l \Lambda S}(r) \mathrm{d} r\right] .
\end{aligned}
$$

\section{The spin-dipole interaction}

The interaction between the electronic-spin magnetic-dipole moments of each atom produces the spin-dipole interaction Hamiltonian

$$
H_{\mathrm{sd}}=V_{\mathrm{p}}(r) \frac{1}{\hbar^{2}}\left[3\left(\mathbf{S}_{1} \cdot \hat{\boldsymbol{r}}\right)\left(\mathbf{S}_{2} \cdot \hat{\boldsymbol{r}}\right)-\mathbf{S}_{1} \cdot \mathbf{S}_{2}\right],
$$

where $\mathbf{S}_{1}$ and $\mathbf{S}_{2}$ are the electronic-spin operators for the two atoms and $\hat{\boldsymbol{r}}$ is a unit vector directed along the internuclear axis. The function $V_{\mathrm{p}}(r)$ is defined by

$$
V_{\mathrm{p}}(r)=-\frac{\beta}{r^{3}} ; \quad \beta=\alpha^{2}\left(\frac{\mu_{\mathrm{e}}}{\mu_{\mathrm{B}}}\right)^{2} E_{\mathrm{h}} a_{0}^{3},
$$

where $\alpha$ is the fine structure constant, $a_{0}$ is the Bohr radius, $\left(\mu_{\mathrm{e}} / \mu_{\mathrm{B}}\right)=1.00115965$ is the electron magnetic moment and $E_{\mathrm{h}}$ is the Hartree energy (=1 a.u.). The perturbation $H_{\text {sd }}$ in (27) can easily be identified with $H_{\mathrm{p}}$ in (15) since

$$
\mathbf{S}_{1} \mathbf{S}_{2}=\frac{1}{2}\left[\mathbf{S S}-\mathbf{S}_{1} \mathbf{S}_{1}-\mathbf{S}_{2} \mathbf{S}_{2}\right]
$$

where $\mathbf{S}=\mathbf{S}_{1}+\mathbf{S}_{2}$ is the operator for the total spin. Therefore in (15), $\lambda=2$ and

$$
\mathbf{T}(2) \equiv \mathcal{S}(2)-\mathcal{S}_{1}(2)-\mathcal{S}_{2}(2) ; \quad \mathbf{U}(2) \equiv \frac{1}{2}(3 \hat{\boldsymbol{r}} \hat{\boldsymbol{r}}-\mathbf{I}),
$$

where $\mathbf{I}$ is the unit dyadic. In (30), $\mathcal{S}(2), \mathcal{S}_{1}(2), \mathcal{S}_{2}(2)$ and $\mathbf{U}(2)$ are irreducible tensors with components

$$
\begin{aligned}
& \mathcal{S}(2 q)=\frac{1}{\hbar^{2}}\left(\frac{4 \pi}{5}\right)^{\frac{1}{2}} S^{2} Y_{2 q}(\hat{\mathbf{S}}) ; \\
& \mathcal{S}_{i}(2 q)=\frac{1}{\hbar^{2}}\left(\frac{4 \pi}{5}\right)^{\frac{1}{2}} S_{i}^{2} Y_{2 q}\left(\hat{\mathbf{S}}_{i}\right) ; \quad i=1,2
\end{aligned}
$$

and

$$
U(2 q)=\left(\frac{4 \pi}{5}\right)^{\frac{1}{2}} Y_{2 q}(\hat{\boldsymbol{r}}) .
$$

In (31) and (32), functions of the type $Y_{2 q}(\hat{\mathbf{x}})$ are spherical harmonics and explicit expressions for $\left\langle S^{\prime}|| \mathbf{T}(2)|| S\right\rangle$ and $\left\langle l^{\prime}|| \mathbf{U}(2) \| l\right\rangle$ are given in (A.3. - (A.5).

\section{Spin-polarized metastable helium atoms}

For the case of metastable helium atoms, $\Lambda=0, S_{i}=1 ; i=1,2$ and so the adiabatic potentials required for the ${ }^{1} \Sigma_{g}^{+}$and ${ }^{5} \Sigma_{g}^{+}$molecular states are the potentials $V_{00}(r)$ and $V_{02}(r)$. Initially the atoms are spin polarized so that $S=2$ and $M_{S}=2$ and 
then collisions take place that produce final states with $S^{\prime}=2, M_{S}^{\prime}=0, \pm 1, \pm 2$ and $S^{\prime}=0, M_{S}^{\prime}=0$. For the ${ }^{5} \Sigma_{g}^{+}$state we use the analytical potential of Stärck and Meyer [16] which has a scattering length of $157 a_{0}$ and supports 15 bound states. For the ${ }^{1} \Sigma_{g}^{+}$potential, we use the results obtained by Müller et al [17] for $r<12 a_{0}$ and for $r \geq 12 a_{0}$, the potential is matched smoothly onto the long-range form $V_{02}(r)-V_{\text {exch }}(r)$ where $V_{\text {exch }}(r)=A \exp (-\gamma r)$ [18, 19]. If the spin polarization is destroyed, there is a high probability of Penning and associative ionization and subsequent loss of atoms from the trap. We model this loss by using a complex optical potential of the form $V_{\Lambda^{\prime} S^{\prime}}(r)=V_{00}(r)-\frac{1}{2} \mathrm{i} \Gamma_{00}(r)$. Two forms for $\Gamma_{00}(r)$ are used; $\Gamma_{\mathrm{M}}(r)$ a least squares fit to the tabulated results in [17] and the simpler form $\Gamma_{\mathrm{GMS}}(r)=0.3 \exp (-r / 1.086)$ of Garrison et al 20] which decreases more rapidly as $r$ increases and does not decrease for small values of $r$.

For this case, the change in energy of the states with $l=0$ is obtained from (26) (28) and (A.6), i.e.

$$
\Delta E_{2}=\frac{2}{5} \beta^{2} \int_{0}^{\infty} F_{k 002}(r) \frac{1}{r^{3}}\left[G_{k 200}(r)+7 G_{k 202}(r)\right] \mathrm{d} r .
$$

In [13], it was shown that since the effective range of the bound-state wave function is typically $10^{3} a_{0}$ to $10^{4} a_{0}$, the wave function $F_{k l \Lambda S}$ for a trap state could be replaced by a free-wave function of the same energy and an excellent value for the energy obtained. Therefore in this application, the energy shifts and widths are calculated using both bound-state $\left(\Delta_{\text {trap }}=1\right)$ and free-wave solutions $\left(\Delta_{\text {trap }}=0\right)$ of (4) for $F_{k 002}$ to test further the validity of the free-wave approximation. Energy shifts and widths are also calculated for trap states with $l=2$.

\section{Numerical calculations}

The unperturbed eigenvalue equation in the form (44) or (17) was solved using the two computational methods described in [13. The first combines the use of quantum defect theory, numerical integration and an iterative procedure (QDT) and in the second a direct numerical solution is obtained using a discrete variable representation (DVR) of the kinetic energy operator and a scaled radial coordinate grid. The DVR method is easily modified to solve (20) for the perturbed functions $G_{k l \Lambda S}(\rho)$. A general real invertible transformation of the radial variable $\rho$ given by

$$
t=u(\rho) ; \quad \rho=u^{-1}(t) \equiv U(t)
$$

is introduced so that (20) becomes

$$
\left[-f^{2} \frac{\mathrm{d}^{2}}{\mathrm{~d} t^{2}} f^{2}+Q(t)\right] \tilde{G}(t)=\tilde{W}(t) \tilde{F}(t),
$$

where

$$
\begin{array}{rlrl}
f(t) & \equiv\left[\frac{\mathrm{d} U}{\mathrm{~d} t}\right]^{-1 / 2} ; & \tilde{F}(t) \equiv \frac{F_{k l \Lambda S}[U(t)]}{f(t)} ; \\
\tilde{G}(t) \equiv \frac{G_{k l^{\prime} \Lambda^{\prime} S^{\prime}}[U(t)]}{f(t)} ; & \tilde{W}(t) \equiv \frac{W[U(t)]}{f(t)}
\end{array}
$$


and

$$
Q(t)=\frac{l^{\prime}\left(l^{\prime}+1\right)}{\rho^{2}}+\rho^{2}+\frac{2 V_{\lambda^{\prime} S^{\prime}}(\rho)}{\hbar \omega}+f^{3} \frac{\mathrm{d}^{2} f}{\mathrm{~d} t^{2}}-4 n^{*} .
$$

The DVR is constructed by using a finite set of basis functions $\left\{\phi_{m}(t)\right\}$ and coordinate points $\left\{t_{m}\right\}$ over the interval $\left[t_{1}, t_{N}\right]$, so that the differential equation (35) is transformed into the matrix eigenvalue equation

$$
\sum_{j=1}^{N}\left[f^{2}\left(t_{i}\right) T_{i j} f^{2}\left(t_{j}\right)+Q\left(t_{i}\right) \delta_{i j}\right] \tilde{G}\left(t_{j}\right)=\tilde{W}\left(t_{i}\right) \tilde{F}\left(t_{i}\right)
$$

where $i=1,2, \ldots N$. The matrix element $T_{i j}$ of the kinetic energy operator $T=-\mathrm{d}^{2} / \mathrm{d} t^{2}$ obtained using a Fourier basis is given in [13] and we choose $\rho_{1} \xi=2 a_{0}$ and $\rho_{N}=15$. The scaling is given by

$$
t=u(\rho)=\left(\frac{\rho}{\zeta}\right)^{1 / p} ; \quad \rho=U(t)=\zeta t^{p}
$$

and we choose $\zeta=20$ and $p=10$ so that about $17 \%$ of the scaled mesh points lie between $\rho_{1}$ and $\zeta$ where the interatomic and spin-dipole interactions are significant. Four to five digit convergence is obtained for the perturbed energies with $N=2000$.

Alternatively, having determined the values of $n^{*}$ for the bound states, (41) and (19) are solved for the correct energies with $\Delta_{\text {trap }}=0$. The previous numerical procedure QDT can be readily modified for this purpose and equations (41) and (19) are integrated numerically using the Numerov algorithm. The solution of (19) contains, in general, a particular integral plus a complementary function which is some linear combination of $F_{l^{\prime}}^{\mathrm{R}}(r)$ and $F_{l^{\prime}}^{\mathrm{I}}(r)$ as defined by (21) with $l \Lambda S$ replaced by $l^{\prime} \Lambda^{\prime} S^{\prime}$. The method, to be labelled as QDTF, is as follows. Equation (41) is integrated outwards from the origin to some $r=r_{\max }$ and $F_{l^{\prime}}^{\mathrm{R}}(r)$ and $\mathrm{d} F_{l^{\prime}}^{\mathrm{R}}(r) / \mathrm{d} r$ are obtained. On matching to solutions that are asymptotically plane waves using methods similar to those described in [13], we obtain $\delta_{l^{\prime}}(k)$. Then $F_{l^{\prime}}^{\mathrm{I}}(r)$ and $\mathrm{d} F_{l^{\prime}}^{\mathrm{I}}(r) / \mathrm{d} r$ are calculated at $r=r_{\max }$, c.f. (21), so that (44) can be integrated inwards to obtain the irregular solution. Integration of (19) requires some care in dealing with the multiples of $F_{l^{\prime}}^{\mathrm{R}}(r)$ and $F_{l^{\prime}}^{\mathrm{I}}(r)$ that build up in the Numerov integration in both the outward and inward directions. In the integration of (19) outwards, multiples of $F_{l^{\prime}}^{\mathrm{R}}(r)$ are removed at each integration step determined so that the function at the current point is zero and this specifies $G_{\text {out }}(r)$. The function $G_{\text {in }}(r)$ is obtained by integrating inwards and a multiple of $F_{l^{\prime}}^{\mathrm{I}}(r)$ is subtracted at the end to make the function zero at the innermost point. Finally, a multiple of $F_{l^{\prime}}^{\mathrm{R}}(r)$ is added to $G_{\text {out }}(r)$ so that the solution matches $G_{\text {in }}(r)$ at $r \approx 6 a_{0}$ and this procedure is very insensitive to the precise choice of matching point. This completes the specification of the solution $G_{k^{\prime} l^{\prime} \Lambda^{\prime} S^{\prime}}(r)$ of the inhomogenous equation (19). It is found that for $r_{\max }=1000 a_{0}$ convergence in the evaluation of the radial integrals in (33) has been obtained correct to three significant figures. 


\section{Results and discussion}

The scaled energy shifts and widths $\Delta n^{*}$ for the five lowest trap states with $l=0$ have been calculated using the DVR method for trapping frequencies $\nu=\omega / 2 \pi$ ranging from $1 \mathrm{kHz}$ to $10 \mathrm{MHz}$. Calculations have also been carried out for frequencies $100 \mathrm{kHz}, 1$ $\mathrm{MHz}$ and $10 \mathrm{MHz}$ using the QDTF approximation and the agreement with the DVR results is very satisfactory. Differences for transitions to the $S^{\prime}=0$ states range from $3 \%$ for the lowest state to $0.4 \%$ for the highest, and at $10 \mathrm{MHz}$ are always $\leq 1 \%$. For transitions to the $S^{\prime}=2$ state the differences are even less, they are $\leq 0.3 \%$ except for the lowest state at $100 \mathrm{kHz}$ where the difference is $2 \%$. It is clear why the QDTF approximation is not so good for the lowest states as the range of $r$ over which the bound-state wave function can be approximated closely by a free wave decreases with decreasing energy. The QDTF approximation has also been used in (26) to investigate widths and shifts for $l=2$ and they prove to be negligible. This is not surprising as the perturbation weights the inner region strongly where the initial wave functions with $l \neq 0$ are very small.

The results shown in table 1 have been obtained using the DVR method. The contributions to $\Delta n^{*}$ of the terms with $S^{\prime}=0,2$ in (33) are also shown, together with the effective quantum number $n^{*}$ and the lifetime $\tau$ given by

$$
\tau=-1 /\left(4 \pi \nu \Im \Delta n^{*}\right) .
$$

The contributions from $S^{\prime}=0$ are sensitive to the form used for $\Gamma_{00}(r)$. In table 1 we also show lifetimes obtained using both $\Gamma_{\mathrm{M}}(r)$ and $\Gamma_{\mathrm{GMS}}(r)$. The energy shifts increase with trapping frequency and $n^{*}$, but the fractional shifts $\Delta n^{*} / n^{*}$ decrease with $n^{*}$ and are of the order $10^{-5}$ to $10^{-7}$. The energy shift arises predominantly from the transitions to $S^{\prime}=2$ states. As expected, the lifetimes $\tau_{\mathrm{GMS}}$ are shorter than the $\tau_{\mathrm{M}}$, as $\Gamma_{\mathrm{GMS}}(r)$ is larger than $\Gamma_{\mathrm{M}}(r)$ for all $r \leq 5 a_{0}$. This dependence suggests that an experimental study of lifetimes as a function of trap frequency could yield an improved knowledge of the potential representing the decay channel.

The decay widths and lifetimes depend strongly on trapping frequency and the lifetimes range from the order of $10^{4} \mathrm{~s}$ for $1 \mathrm{kHz}$ traps to the order of $15 \mathrm{~ms}$ for 10 $\mathrm{MHz}$ traps. The lifetimes for the lower frequencies are greater than both the metastable helium lifetime of $8000 \mathrm{~s}$ and the typical lifetimes of experimental traps which are of the order of seconds [2]. Over the range of frequencies investigated the real and imaginary parts of $\Delta n^{*}$ can be quite closely fitted to the analytic form

$$
\Delta n^{*}=-A \sqrt{x} /\left(1+B x+C x^{2}+D x^{3}\right) ; \quad x=\nu n^{*},
$$

where $\nu$ is in units of $\mathrm{MHz}$ and

$$
\begin{array}{ll}
A=4.0144 \times 10^{-6} ; & B=1.8806 \times 10^{-2} ; \\
C=3.0740 \times 10^{-4} ; & D=-3.3204 \times 10^{-6}
\end{array}
$$

for $\Re \Delta n^{*}$. For $\Im \Delta n^{*}$

$$
A=1.7294 \times 10^{-7} ; \quad B=1.7145 \times 10^{-2} ;
$$




$$
C=3.3987 \times 10^{-4} ; \quad D=-3.7630 \times 10^{-6}
$$

and the parametric fit to the lifetimes is then directly obtained from (40), (41) and (43).

In conclusion, second-order perturbation theory with a rather general form for the perturbation and valid for states with non-zero angular momentum has been developed here. These results are important and have been obtained so that the analysis can be directly applied to other perturbations in future.

\section{Appendix}

The book on Angular Momentum by Edmonds 21] is the basic reference for the notation, equation numbers and tables quoted below. The Wigner-Eckhart theorem, see (5.4.1), defines the reduced matrix elements $\left\langle j^{\prime}|| \mathbf{X}(\lambda) \| j\right\rangle$ using the relation

$$
\left\langle j^{\prime} m_{j}^{\prime}|X(\lambda q)| j m_{j}\right\rangle=(-1)^{j^{\prime}-m_{j}^{\prime}}\left\langle j^{\prime}\|\mathbf{X}(\lambda)\| j\right\rangle\left(\begin{array}{ccc}
j^{\prime} & \lambda & j \\
-m_{j}^{\prime} & q & m_{j}
\end{array}\right),
$$

where $\left(\begin{array}{ccc}a & b & c \\ d & e & f\end{array}\right)$ is a $3-j$ symbol and the average over the degenerate initial states $|l m\rangle$ is carried out using

$$
(2 j+1)^{-1} \sum_{m_{j}^{\prime} m_{j}}\left(\begin{array}{ccc}
j^{\prime} & \lambda & j \\
-m_{j}^{\prime} & q^{\prime} & m_{j}
\end{array}\right)\left(\begin{array}{ccc}
j^{\prime} & \lambda & j \\
-m_{j}^{\prime} & q & m_{j}
\end{array}\right)=\left[(2 j+1)\left(2 j^{\prime}+1\right)\right]^{-1} \delta_{q^{\prime} q},
$$

see (3.7.8). The states $\left|S M_{S}\right\rangle$ and $\left|S_{i} M_{S_{i}}\right\rangle$ are eigenvectors of the operators $\mathbf{S}^{2}, S_{z}, \mathbf{S}_{i}^{2}$, and $S_{i z}, i=1,2$. Therefore using (A.1), (4.6.3) and table 2, the reduced matrix elements required are given by

$$
\begin{aligned}
& \left\langle S_{1}^{\prime} S_{2}^{\prime} S^{\prime}\|\mathcal{S}(2)\| S_{1} S_{2} S\right\rangle=\frac{1}{4}[(2 S+3)(2 S+2)(2 S+1)(2 S)(2 S-1)]^{\frac{1}{2}} \delta_{S^{\prime} S} ; \\
& \left\langle S_{i}^{\prime} \mid \mathcal{S}_{i}(2) \| S_{i}\right\rangle=\frac{1}{4}\left[\left(2 S_{i}+3\right)\left(2 S_{i}+2\right)\left(2 S_{i}+1\right)\left(2 S_{i}\right)\left(2 S_{i}-1\right)\right]^{\frac{1}{2}} \delta_{S_{i}^{\prime} S_{i}} ; \quad i=1,2
\end{aligned}
$$

and

$$
\left|\left\langle l^{\prime}|| \mathbf{U}(2)|| l\right\rangle\right|^{2}=(2 l+1)\left(2 l^{\prime}+1\right)\left|\left(\begin{array}{ccc}
l^{\prime} & 2 & l \\
0 & 0 & 0
\end{array}\right)\right|^{2} .
$$

Finally, using (7.1.7) and (7.1.8), the reduced matrix elements of the spin operators $\mathcal{S}_{1}$ and $\mathcal{S}_{2}$ in the coupled representation are given by

$$
\begin{aligned}
& \left\langle S_{1}^{\prime} S_{2}^{\prime} S^{\prime}\left\|\mathcal{S}_{1}(2)\right\| S_{1} S_{2} S\right\rangle=(-1)^{S_{1}^{\prime}+S_{2}+S}\left[(2 S+1)\left(2 S^{\prime}+1\right)\right]^{\frac{1}{2}}\left\{\begin{array}{ccc}
S_{1}^{\prime} & S^{\prime} & S_{2} \\
S & S_{1} & 2
\end{array}\right\} \\
& \quad \times\left\langle S_{1}^{\prime}\left\|\mathcal{S}_{1}(2)\right\| S_{1}\right\rangle \delta_{S_{2}^{\prime} S_{2}} ; \\
& \left\langle S_{1}^{\prime} S_{2}^{\prime} S^{\prime}\left\|\mathcal{S}_{2}(2)\right\| S_{1} S_{2} S\right\rangle=(-1)^{S_{1}+S_{2}+S^{\prime}}\left[(2 S+1)\left(2 S^{\prime}+1\right)\right]^{\frac{1}{2}}\left\{\begin{array}{ccc}
S_{2}^{\prime} & S^{\prime} & S_{1} \\
S & S_{2} & 2
\end{array}\right\} \\
& \quad \times\left\langle S^{\prime} 2\left\|\mathcal{S}_{2}(2)\right\| S_{2}\right\rangle \delta_{S_{1}^{\prime} S_{1}},
\end{aligned}
$$

where $\left\{\begin{array}{lll}a & b & c \\ d & e & f\end{array}\right\}$ is a $6-j$ symbol.

The following important special cases are easily derived from (A.1) - (A.5) and tables 2 and 5 :

$|\langle 0|| \mathbf{T}(2) \| 2\rangle|^{2}=10 ; \quad|\langle 2|| \mathbf{T}(2)|| 2\rangle|^{2}=70 ; \quad|\langle 2|| \mathbf{U}(2) \| 0\rangle|^{2}=1$. 


\section{References}

[1] Greiner M, Mandel O, Esslinger T, Hánsch T W and Bloch I 2002 Nature 415 39-44

[2] Müller-Seydlitz T, Hartl M, Brezger B, Hänsel H, Keller C, Schnetz A, Spreeuw R J C, Pfau T and Mlynek J 1997 Phys. Rev. Lett. 78 1038-41

[3] Brennen G K, Caves C M, Jessen P S and Deutsch I H 1999 Phys. Rev. Lett. 82 1060-3

[4] Jaksch D, Briegel H-J, Cirac J I, Gardiner C W and Zoller P 1999 Phys. Rev. Lett. 82 1975-8

[5] Tiesinga E, Williams C J, Mies F H and Julienne P S 2000 Phys. Rev. A 61063416

[6] Bolda E L, Tiesinga E and Julienne P S 2002 Phys. Rev. A 66013403

[7] Blume D and Greene C H 2002 Phys. Rev. A 65043613

[8] Pereira Dos Santos F, Léonard J, Wang Junmin, Barrelet C J, Perales F, Rasel E, Unnikrishnan C S, Leduc M and Cohen-Tannoudji C 2001 Phys. Rev. Lett. 86 3459-62

[9] Robert A, Sirjean O, Browaeys A, Poupard J, Nowak S, Boiron D, Westbrook C I and Aspect A 2001 Science 292 461-4

[10] Sirjean O, Seidelin S, Viana Gomes J, Boiron D, Westbrook C I, Aspect A and Shlyapnikov G V 2002 Phys. Rev. Lett. 89220406

[11] Seidelin S, Sirjean O, Viana Gomes J, Boiron D, Westbrook C I and Aspect A 2002 e-print arXiv:cond-mat/0211112

[12] Peach G, Whittingham I B and Beams T J 2002 Interactions of Cold Atoms and Molecules ed P Soldán, M T Cvitaš, J M Hutson and C S Adams (Daresbury: Collaborative Computational Project on Molecular Quantum Dynamics (CCP6)) p 85-8

[13] Peach G, Whittingham I B and Beams T J 2004 e-print arXiv:physics/0212003 Phys. Rev. A to be published

[14] Dalgarno A and Lewis J T 1955 Proc. R. Soc. A 233 70-4

[15] Abramowitz M and Stegun I A 1965 Handbook of Mathematical Functions (New York NY: Dover)

[16] Stärck J and Meyer W 1994 Chem. Phys. Lett. 225 229-32

[17] Müller M W, Merz A, Ruf M-W, Hotop H, Meyer W and Movre M 1991 Z. Phys. D: At., Mol. Clusters 21 89-112

[18] Venturi V, Whittingham I B, Leo P J and Peach G 1999 Phys. Rev. A 60 4635-46

[19] Leo P J, Venturi V, Whittingham I B and Babb J F 2001 Phys. Rev. A 64042710

[20] Garrison B J, Miller W H and Schaefer H F 1973 J. Comput. Phys. 59 3193-9

[21] Edmonds A R 1974 Angular Momentum in Quantum Mechanics 2nd edn (Princeton NJ: Princeton University Press) 
Tables and table captions

Table 1. Effective quantum numbers $n^{*}=E /(2 \hbar \omega)$, shifts $\Delta n^{*}$ (in units of $10^{-5}$ ) and state lifetimes $\tau_{\mathrm{M}}$ for harmonic traps with frequencies ranging from $1 \mathrm{kHz}$ to $10 \mathrm{MHz}$. Also shown are the lifetimes $\tau_{\mathrm{GMS}}$ for comparison. Numbers in parentheses denote powers of 10 .

\begin{tabular}{|c|c|c|c|c|c|c|}
\hline$n_{r}^{\prime}$ & $n^{*}$ & $\Delta n^{*}\left(S^{\prime}=0\right)$ & $\Delta n^{*}\left(S^{\prime}=2\right)$ & $\Delta n^{*}($ Total $)$ & $\tau_{\mathrm{M}}(\mathrm{s})$ & $\tau_{\mathrm{GMS}}(\mathrm{s})$ \\
\hline \multicolumn{7}{|c|}{$\nu=1 \mathrm{kHz}$} \\
\hline 0 & 0.7521 & $-0.0007-0.0005 \mathrm{i}$ & -0.0105 & $-0.0112-0.0005 \mathrm{i}$ & $1.65(4)$ & $1.42(4)$ \\
\hline 1 & 1.7531 & $-0.0010-0.0007 \mathrm{i}$ & -0.0158 & $-0.0168-0.0007 \mathrm{i}$ & $1.10(4)$ & $9.47(3)$ \\
\hline 2 & 2.7539 & $-0.0013-0.0009 \mathrm{i}$ & -0.0197 & $-0.0210-0.0009 \mathrm{i}$ & $8.83(3)$ & $7.58(3)$ \\
\hline 3 & 3.7546 & $-0.0015-0.0011 \mathrm{i}$ & -0.0229 & $-0.0244-0.0011 \mathrm{i}$ & $7.57(3)$ & $6.50(3)$ \\
\hline 4 & 4.7551 & $-0.0017-0.0012 \mathrm{i}$ & -0.0258 & $-0.0275-0.0012 \mathrm{i}$ & $6.73(3)$ & $5.78(3)$ \\
\hline \multicolumn{7}{|c|}{$\nu=10 \mathrm{kHz}$} \\
\hline 0 & 0.7566 & $-0.0022-0.0015 \mathrm{i}$ & -0.0335 & $-0.0356-0.0015 \mathrm{i}$ & $5.20(2)$ & $4.47(2)$ \\
\hline 1 & 1.7599 & $-0.0032-0.0023 \mathrm{i}$ & -0.0500 & $-0.0532-0.0023 \mathrm{i}$ & $3.48(2)$ & $2.99(2)$ \\
\hline 2 & 2.7624 & $-0.0040-0.0029 \mathrm{i}$ & -0.0623 & $-0.0663-0.0029 \mathrm{i}$ & $2.79(2)$ & $2.39(2)$ \\
\hline 3 & 3.7644 & $-0.0047-0.0033 \mathrm{i}$ & -0.0726 & $-0.0773-0.0033 \mathrm{i}$ & $2.39(2)$ & $2.05(2)$ \\
\hline 4 & 4.7662 & $-0.0053-0.0037 \mathrm{i}$ & -0.0816 & $-0.0869-0.0037 \mathrm{i}$ & $2.13(2)$ & $1.83(2)$ \\
\hline \multicolumn{7}{|c|}{$\nu=100 \mathrm{kHz}$} \\
\hline 0 & 0.7711 & $-0.0069-0.0049 \mathrm{i}$ & -0.1073 & $-0.1142-0.0049 \mathrm{i}$ & $1.62(1)$ & $1.39(1)$ \\
\hline 1 & 1.7815 & $-0.0102-0.0073 \mathrm{i}$ & -0.1588 & $-0.1691-0.0073 \mathrm{i}$ & $1.09(1)$ & 9.37 \\
\hline 2 & 2.7893 & $-0.0127-0.0091 \mathrm{i}$ & -0.1971 & $-0.2098-0.0091 \mathrm{i}$ & 8.78 & 7.54 \\
\hline 3 & 3.7957 & $-0.0147-0.0105 \mathrm{i}$ & -0.2288 & $-0.2435-0.0105 \mathrm{i}$ & 7.56 & 6.50 \\
\hline 4 & 4.8014 & $-0.0165-0.0118 \mathrm{i}$ & -0.2562 & $-0.2727-0.0118 \mathrm{i}$ & 6.75 & 5.80 \\
\hline \multicolumn{7}{|c|}{$\nu=1 \mathrm{MHz}$} \\
\hline 0 & 0.8181 & $-0.0225-0.0160 \mathrm{i}$ & -0.3493 & $-0.3718-0.0160 \mathrm{i}$ & $4.96(-1)$ & $4.26(-1)$ \\
\hline 1 & 1.8502 & $-0.0318-0.0229 \mathrm{i}$ & -0.4960 & $-0.5277-0.0229 \mathrm{i}$ & $3.48(-1)$ & $2.99(-1)$ \\
\hline 2 & 2.8738 & $-0.0382-0.0275 \mathrm{i}$ & -0.5967 & $-0.6349-0.0275 \mathrm{i}$ & $2.89(-1)$ & $2.48(-1)$ \\
\hline 3 & 3.8930 & $-0.0430-0.0311 \mathrm{i}$ & -0.6740 & $-0.7170-0.0311 \mathrm{i}$ & $2.56(-1)$ & $2.19(-1)$ \\
\hline 4 & 4.9096 & $-0.0469-0.0340 \mathrm{i}$ & -0.7362 & $-0 . .7831-0.0340 \mathrm{i}$ & $2.34(-1)$ & $2.01(-1)$ \\
\hline \multicolumn{7}{|c|}{$\nu=10 \mathrm{MHz}$} \\
\hline 0 & 0.9703 & $-0.0645-0.0469 \mathrm{i}$ & -1.0145 & $-1.0791-0.0469 \mathrm{i}$ & $1.70(-2)$ & $1.46(-2)$ \\
\hline 1 & 2.0509 & $-0.0720-0.0533 \mathrm{i}$ & -1.1468 & $-1.2188-0.0533 \mathrm{i}$ & $1.49(-2)$ & $1.28(-2)$ \\
\hline 2 & 3.1040 & $-0.0732-0.0550 \mathrm{i}$ & -1.1783 & $-1.2516-0.0550 \mathrm{i}$ & $1.45(-2)$ & $1.24(-2)$ \\
\hline 3 & 4.1442 & $-0.0725-0.0551 \mathrm{i}$ & -1.1777 & $-1.2502-0.0551 \mathrm{i}$ & $1.44(-2)$ & $1.24(-2)$ \\
\hline 4 & 5.1767 & $-0.0710-0.0546 \mathrm{i}$ & -1.1648 & $-1.2358-0.0546 \mathrm{i}$ & $1.46(-2)$ & $1.25(-2)$ \\
\hline
\end{tabular}

